\title{
Ship electromotive system with common DC buses
}

\author{
Fedor Gelver ${ }^{1, *}$, Veniamin Samoseiko ${ }^{1}$, and Alexandr Saushev ${ }^{1}$ \\ ${ }^{1}$ Admiral Makarov State University of Maritime and Inland Shipping, 5/7, Dvinskaya Str., Saint \\ Petersburg, 198035, Russia
}

\begin{abstract}
The universal structure of the ship's electromotive system with common DC (direct current) buses is proposed. It is shown that this structure allows: improving operational, energy, environmental and massdimensional characteristics of the motive system; increasing the reliability and survivability of the ship's electric power system; braking propellers with the transfer of braking energy to consumers of its own needs or to the energy storage; redistributing energy between propulsion electric machines with the dissipation of braking energy in the working environment; providing fuel economy in the range of $12 \div 15 \%$ depending on the modes of operation of the ship in comparison with the known structures of electric propulsion systems. Analytical dependences that allow choosing the voltage level of the electric generator and coordinating it with the level of nominal voltages on the propeller motors and other load connected to the voltage inverters are obtained. A function for the analytical description of the limiting reversible mechanical characteristic of the propeller is synthesized. The mathematical description of the braking and reverse modes of the propulsion electric plant is given. Synthesis of algorithms of control of propulsion electric plant with two propellers at braking and reverse is carried out. The time diagrams of the torque, speed and power changes at each of the propellers for the proposed algorithm of braking and reverse of the propeller motors are presented.
\end{abstract}

\section{Introduction}

Nowadays, in the modern world shipbuilding, there is a steady tendency to build ships with partial and full electric propulsion systems [1-4], as well as fully electric ships, which use only batteries as a source of energy [5-7]. The choice of such structures of ship's electromotive system is due to the desire to reduce harmful emissions into the environment [8]. However, such innovative ships currently have a very limited range of travel and, as a consequence, a small sailing area. They are mainly used as tugs in the waters of ports or as ferries and pleasure craft. The main requirement for the design of ships with electric propulsion systems is to provide higher performance and energy characteristics compared to systems using direct drive propellers from primary heat engines. In recent years, the requirements for environmental indicators have become stricter [8]. The wide application of

\footnotetext{
*Corresponding author: ep-gumrf@bk.ru
} 
electric propulsion systems on various types of ships is limited by the large weight, dimensions, cost, as well as low reliability and complexity of the electrical equipment used.

There is a wide variety of structures of ship's electromotive system, made on direct and alternating current [9-13]. Each of these structures has its advantages and disadvantages, has the right to exist, and can be applied in practice depending on the requirements that apply to a particular system of electric propulsion. It should be noted that in recent years, hybrid electromotive systems [14-18] have gained popularity and development, which can significantly save fuel, as well as modular electromotive systems with minimal weight and size characteristics [19-22].

Thus, the task of synthesis of universal structure of ship's electromotive system characterized by high quality indicators and meeting modern development trends is actual.

\section{Materials and methods}

The paper deals with the structure of ship's electromotive system with common DC busses (figure 1). This solution eliminates a number of shortcomings inherent in the structures of electric propulsion systems both direct and alternating current. The proposed structure allows to increase energy efficiency, reliability, increase the load factor of primary thermal motors of the electromotive system and, as a consequence, to provide fuel economy and increase the technical resource of the power system elements. The versatility of the proposed solution lies in the fact that the structure under consideration can be used to power any type of AC (alternating current) propulsion motors with a wide range of speed control of propellers and consumers of its own needs. A distinctive feature of the ship's electromotive system with common DC buses is that the AC electric generators operate on voltage rectifiers whose outputs form common DC buses. At the same time, all power consumers on the ship are powered by these common buses. To the common buses of the DC link, voltage inverters of the propeller motors can be connected, through a matching electric converter for own needs and an electric energy storage, which will ensure high dynamic characteristics of the ship's electromotive system and bring the total installed power of the primary heat engines to the total power of the ship's consumers.

The advantage of the proposed solution is also that the sources of electric energy through voltage rectifiers operate on common DC buses. In this case, there is no need for commissioning and decommissioning of generator sets, including its accurate synchronization in frequency and phase, and primary heat engines can be configured for optimal operation with maximum efficiency. In addition, high-frequency generator sets can be used, which have a much smaller weight and dimensions compared to known technical solutions. 


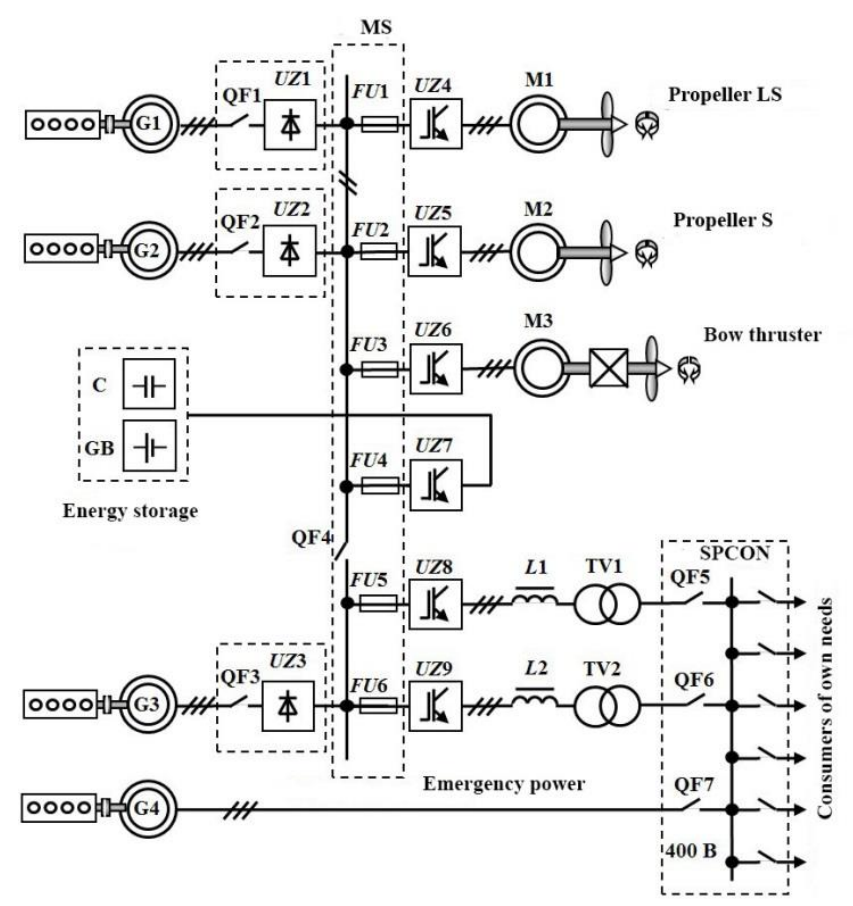

Fig. 1. Structural scheme of a propulsion electric power plant with common DC buses

\section{Selection of voltage value of electric generators}

For the purpose of coordination of voltage levels of generators and loadings the dependences allowing to choose the required voltage level of the synchronous generator and to coordinate it with voltages on the propeller electric motor and on loading of inverters are analytically received. It should be borne in mind that, as a rule, the rated voltage of the generator is selected by $5 \%$ more than the rated voltage of the load. The figure 2 shows the dependences of the relative effective value of the phase electromotive force (EMF) of the generator under rated load as a function of the power coefficient (figure 2 a) and the dependence of the relative effective value of the main harmonic of the phase voltage on the inverter load as a function of the nominal value of the generator power coefficient (figure 2 b). All values in figure 2 are represented in relative units.

a)

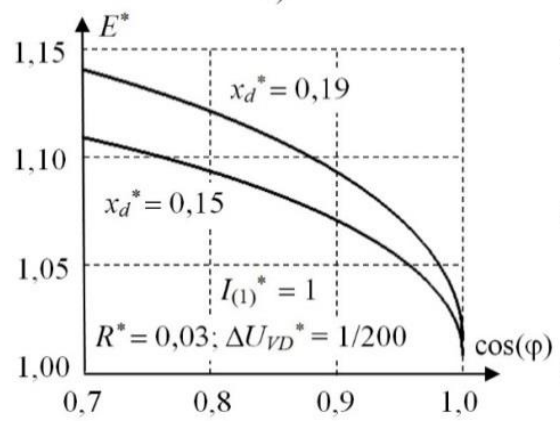

b)

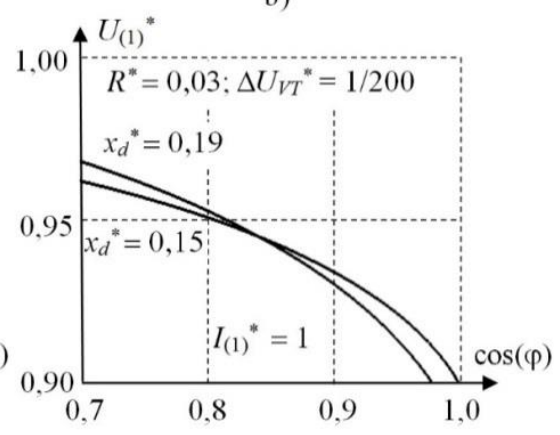

Fig. 2. Graphs of dependences on the power coefficient of the generator under rated load: a) the relative effective value of the phase EMF of the generator; $b$ ) the relative effective value of the main harmonic of the phase voltage on the inverter load 
From the graphs shown in figure 2 it follows that in order to obtain a relative nominal voltage $U_{(1)}{ }^{*} \quad 10.95$ at the load, it is necessary that the generator have a nominal power factor of $0.8 \div 0.82$. If the nominal power factor of the generator is higher than $0.8 \div 0.82$, the so-called "remodulation" mode should be used to provide the voltage at the load $U_{(1)}{ }^{*} \mid 0.95$ when generating the voltage by the inverter. This mode allows you to raise the voltage in $4 / \pi$ times. However, the use of such a mode is undesirable, since in this case the harmonic composition of the synthesized voltage on the load of voltage inverters deteriorates. The excitation control system of generator sets must maintain a constant voltage on the common buses of the DC link.

\section{Design Features of the ship's electromotive system with common DC buses}

When designing the structure of ship's electromotive system with common DC buses, it is necessary to take into account that each source and each consumer of electric energy must contain its own switching and protective apparatus. The protective equipment shall be selected in such a way that, in the event of an emergency, only the source or consumer that caused the emergency is disconnected from the common DC buses. In this case, the efficiency and survivability of the remaining part of the ship's electric power system is maintained. There is also no need for additional protection of DC buses. At the same time, it is necessary to consider that buses of a link of a direct current in this power station have to be executed structurally so that on it's the possibility of occurrence of short circuits was excluded.

The number of generator sets in the proposed structure can be any. Their selection shall be made on the basis of the modes of operation of the ship, the most frequently used modes of electric power installation, and loading of the generators. As electric converters of propulsion electric motors in the scheme voltage inverters which in the structure already contain the accumulative capacitor of a direct current link are established. At the input of each voltage inverter, a protective device, such as a fast-acting fuse, is installed (figure 1), which eliminates the occurrence of an emergency situation on the remaining loads connected to the common buses of the DC link. Any type of AC electric machines can be installed as electric motors.

In order to improve the energy efficiency of the propulsion electric power plant, voltage inverters with matching transformers are connected to the common buses of the DC link to supply auxiliary consumers and consumers of its own needs, and an intermediate energy storage with an individual matching electric converter is connected.

The proposed structure makes it possible to implement various control algorithms that allow to increase energy efficiency and exclude brake circuits from the equipment of the ship's electric power system. Such algorithms include algorithms for reloading the main primary heat engines for energy storage on the drive and algorithms that implement the useful use of braking energy of propeller motors.

For the nominal load of the main primary heat engines the control system electrical power plant determines the value of the power consumed by the propeller motors and consumers its own needs, and includes such a number of the main primary heat engines and electric generators to the total installed capacity of electric generators is equal or slightly more power consumed by all loads. Additional loading of the main primary heat engines and electric generators is carried out by charging the energy storage device through a matching electric converter. This ensures the nominal load of the main primary heat engines and electric generators, which ensures their economical operation. The energy stored in the energy storage is used to provide the required dynamics in the acceleration or start-up modes of the propulsion electric plant, when it is necessary to provide a large 
amount of energy to the propulsion electric motors in the dynamic modes of operation for a short time. The energy storage device shall be selected in such a way as to provide the required acceleration and deceleration dynamics of the ship. Accordingly, in such an electric power plant, the installed capacity of the main primary heat engines and electric generators is equal to the installed capacity of all electricity consumers on the ship.

In the braking modes of the propulsion unit, the energy recovered from the propulsion motors during braking will be consumed to supply consumers with its own needs. In this case, the energy flow will be directed along the circuit from the propeller motors through the voltage inverters to the common DC buses and then through the voltage inverter, the matching voltage transformer to the secondary power supply board and the power supply of consumers for its own needs (figure 1). Unmanaged voltage rectifiers in this mode are in a locked state, since the voltage on the common DC buses is greater than the voltage generated by the electric generators. Thus, in the modes of energy recovery from the propeller motors, the energy from the main primary heat engines and electric generators is not consumed. The inverter of voltage of consumers of own needs works in the mode of stabilization of level and frequency of voltage on buses of secondary power supply, providing power supply of consumers of own needs with voltage with demanded indicators of quality. If the consumers of its own needs are not able to accept the incoming amount of electricity, electricity is discharged to the energy storage device. This occurs via a circuit from the propeller motors through voltage inverters to common DC buses and, through a matching energy converter, to an energy storage device (figure 1).

\section{$5 \quad$ Braking of electric propeller with two propellers}

If the consumers of its own needs and the energy storage can not take over the recovered electricity, braking will be carried out by the redistribution of energy between the propeller motors. The figure 3 shows the direction of energy flows during braking and reverse propeller electric drive with two propellers.

Management of frequency of rotation of the rotor shaft of the electric motor is connected with the control of the electromagnetic torque. This relationship follows directly from the basic equation of motion presented in operator form and written in relative units:

$$
T_{\text {mech }} \cdot p \omega^{*}=M_{m o v}{ }^{*}-M_{\mathrm{s}}{ }^{*}
$$

where $T_{\text {mech }}=J \cdot b_{\mathrm{b}} / M_{\mathrm{b}}-$ mechanical time constant, $p=\frac{d}{d t}-$ differential operator, $J-$ moment of inertia of the mechanism, $\cdot \omega_{\mathrm{b}}-$ the base speed value, $M_{\mathrm{b}}-$ the basic value of the moment.

For the analytical description of the limiting reversible mechanical characteristic of a propeller in relative units, a function is synthesized with the help of a canonical polynomial, which has the following form:

$$
M_{s}(\omega)=-0,4-0,256 \cdot \omega-7,194 \cdot \omega^{2}+0,544 \cdot \omega^{3}+27,497 \cdot \omega^{4}-0,021 \cdot \omega^{5}-43,968 \cdot \omega^{6}+24,798 \cdot \omega^{7}
$$

The graph of the obtained function is shown in figure 4 . This approximation most accurately describes the limit reversible mechanical characteristic obtained empirically. 


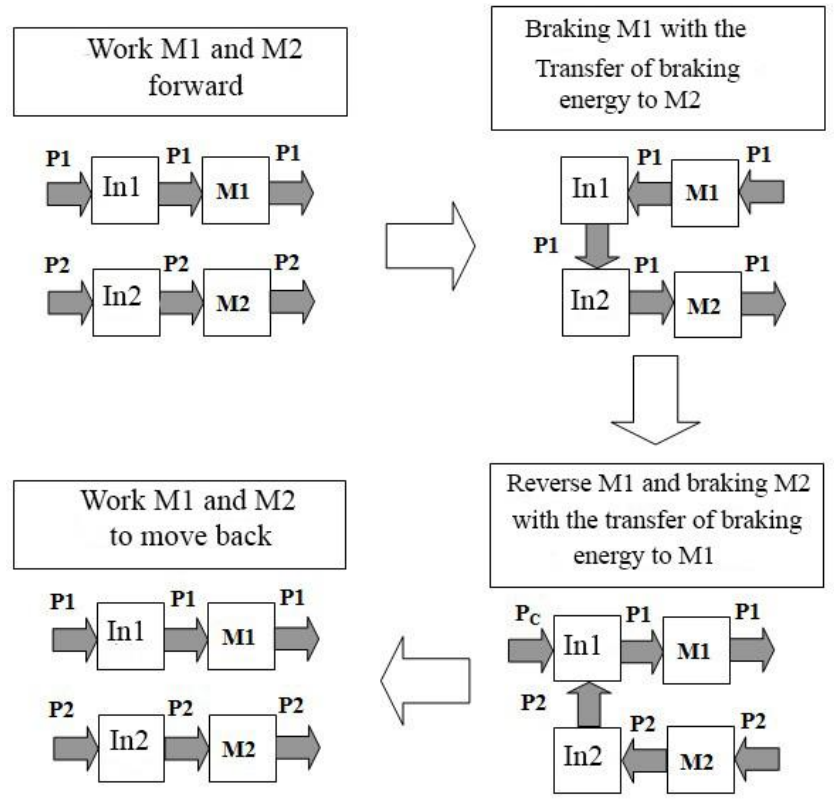

Fig. 3. The direction of energy flows when braking a propeller electric installation with two propellers

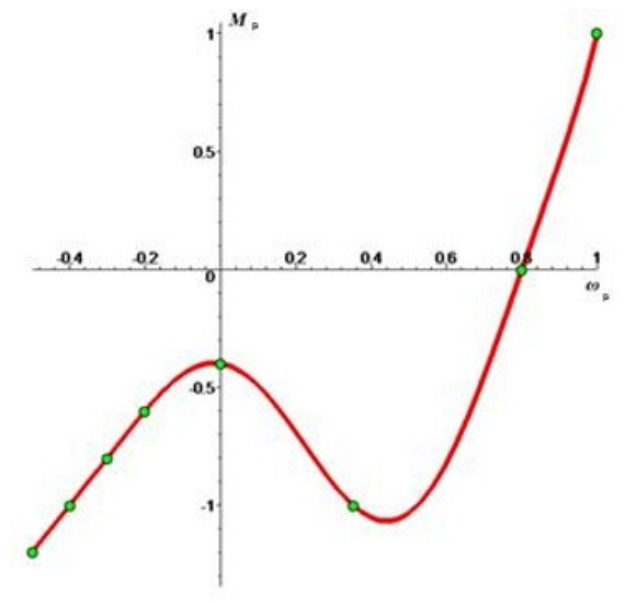

Fig. 4. Ultimate reversible mechanical characteristic of the propeller $M_{p}=f\left(\omega_{p}\right)$

Taking into account the fact that the average time constant of electric motors is about $0.3 \mathrm{~s}$, we take the total time constant of the propeller and the propeller motor equal to $1 \mathrm{~s}\left(T_{\text {mech }}=\right.$ $1 \mathrm{~s})$. Therefore, in the simulation, all processes will be reflected in seconds.

Let us make an analytical description of the process of braking and reversing the propeller according to the proposed algorithm in a propeller electric installation containing two screws (propellers) with an electric drive assembled according to the scheme shown in figure 1.

Let's write down the equations of motion for the first and second propeller

$$
\begin{aligned}
& M_{i 1}=M_{m o v 1}-M_{s 1}=T_{m e c h 1} \frac{d \omega_{1}}{d t} \\
& M_{i 2}=M_{m o v 2}-M_{s 2}=T_{m e c h 2} \frac{d \omega_{2}}{d t}
\end{aligned}
$$


We introduce restrictions on the maximum possible value of the electromagnetic moment developed by the propeller motors, equal to the value $\max \left|M_{\operatorname{mov} 1}\right|=\max \left|M_{\text {mov } 2} \quad\right|=1.2$.

Using the initial conditions $\omega_{1}(0)=1, M_{\operatorname{mov} 1}=-1,2$, and substituting the expression (1) in the formula (2), we obtain the graph of the function $\omega_{1}(t)$ presented in figure 5 . The power on the propeller motor M1 (PEM1) is equal to $P_{\text {mov } 1}(t)=M_{\text {mov } 1} \cdot \omega_{1}(t)$.

The graph of the function $P_{\text {movl }}(t)$ is shown in figure5. Note that the negative value $P_{\text {movl }}(t)$ characterizes the generator mode of operation of the PEM1 and the propeller hydroturbine mode, and the positive value $P_{\text {movl }}(t)$ characterizes the motor mode of operation of the PEM1, in which the propeller creates a stop at the reverse of the PEM1.

Using the condition of equality of powers $P_{\text {movl }}(t)=P_{\text {mov }}(t)$, the value of the electromagnetic moment of the propeller motor PEM2 can be written in the following form:

$$
M_{\text {mov } 2}(t)=\frac{M_{m o v 1} \cdot \omega_{1}(t)}{\omega_{2}(t)}
$$

This equality is valid if there are no losses in electromechanical and electrical converters of the propeller electric installation.

Using the initial conditions $\omega_{2}(0)=1$ and the solution of equation (3) with respect to the electromagnetic moment (4), we obtain the dependence $\omega_{2}(t)$, the graph of which is shown in figure 5 .

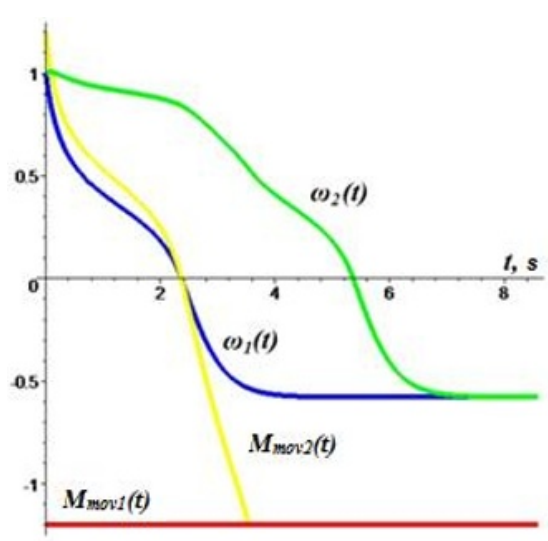

Fig. 5. Dependencies $\omega_{1}(t), M_{\text {movl }}(t), \omega_{2}(t), M_{\text {mov } 2}(t)$ at braking and reverse of propulsion electric unit

Substituting the results of the solution (3) with respect $\omega_{2}(t)$ to the formula (4), we obtain a description of the required change in the value of the electromagnetic moment $M_{\text {mov } 2}(t)$ of the motor PEM2 (figure 5).

Power on the shafts PEM1 and PEM2 are determined according to the expressions

$$
\begin{aligned}
& P_{\text {mov } 1}(t)=M_{\text {mov } 1} \cdot \omega_{1}(t) \\
& P_{\text {mov } 2}(t)=M_{\text {mov } 2} \cdot \omega_{2}(t)
\end{aligned}
$$

The figure 6 shows the graphs of time dependences of capacities on shafts of propeller electric motors PEM 1 and PEM 2 are given. From these graphs it follows that up to a certain point in time (before entering $M_{\operatorname{mov} 2}=-1,2$ to the restriction mode, in which $M_{\text {mov } 2}=-1,2$ ) the sum of the capacities on the shaft PEM 1 and PEM 2 is zero. In this case, there is no need to reset the braking energy to any of the consumers. After reaching the condition $M_{\text {mov } 1}=-1,2$, there is a power take - off from the voltage inverters, necessary for the operation of PEM 1 and PEM 2 on the reverse. 


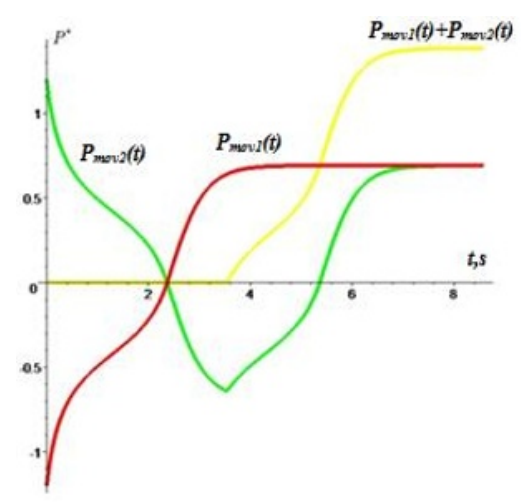

Fig. 6. Dependencies $P_{\text {mov } 1}(t), P_{\text {mov } 2}(t), P_{\text {movl }}(t)+P_{\text {mov } 2}(t)$ at braking and reverse of propeller

The considered processes describe only the processes occurring in the propeller, but do not reflect the processes of stopping and reversing the ship, although these processes affect them.

\section{Discussion of results}

Summing up, it can be concluded that the proposed structure of the propulsion electric power plant with common DC buses is characterized by simplicity of circuit implementation, a minimum number of elements of the power channel and allows the exchange of energy between consumers and the energy storage through intermediate DC link buses. This structure allows to approximate the installed power of primary heat engines and generator sets to the installed power of propeller motors, to use high-frequency generator sets and to load the used energy source under the nominal value without using it idling. This, in turn, will reduce the weight and size and increase the energy performance of the entire power plant. The advantage of the proposed structure is the possibility of increasing the energy efficiency of the propulsion system, reducing the weight and size characteristics and ensuring the specified values of the reliability and survivability of the entire ship's electric power system.

An important advantage of a propulsion electric power plant with common DC buses is the ability to brake and reverse in the presence of several propellers without using a special brake module with the transfer of braking energy to the energy storage device, or to power consumers of its own needs, or not its dispersion in the working environment-water. The proposed system is universal, has "flexibility" and allows to implement various algorithms for controlling the power plant and propulsion electric drives with the ability to control the flow of energy between the propulsion motors, the drive, auxiliary consumers and consumers of its own needs. A distinctive feature of the plant is high environmental friendliness and low amount of polluting emissions into the environment. According to the calculated data, the proposed structure of the propulsion electric power plant will allow for fuel savings in the range of $12 \div 15 \%$ depending on the operating modes in comparison with the known structures of electric propulsion systems of ships.

\section{Conclusion}

A stable trend of the modern stage of shipbuilding development is the increasingly widespread introduction of partial and full electric propulsion systems. This is due to the known advantages of electric energy compared to other types of energy. The performed 
analysis showed that the known block diagrams of propulsion electric power plants differ in a wide variety, however, they all have certain disadvantages that hinder its wide application. The paper considers the structural scheme of the propulsion electric power plant with common DC buses, which is fundamentally different from the known technical solutions, is universal and allows, as shown by the studies, to increase the most important quality indicators characterizing both its operation and the operation of the ship's electric power system as a whole. The obtained mathematical models made it possible to study the dynamic modes of operation of propulsion electric power plants with common DC buses and to quantify the advantage of the proposed solution in comparison with known technical solutions.

\section{References}

1. E. Skjong, R. Volden, E. Rødskar, M.M. Molinas Cabrera, T.A. Johansen, J. Cunningham, Past, Present and Future Challenges of the Marine Vessel's Electrical Power System, (2016).

2. J. Emblemsvag, IEEE Electrific. Mag. 5 (3) 4-9. (2017)

3. E. Skjong, E. Rødskar, M.M. Molinas Cabrera, T.A. Johansen, Joseph Cunningham, The Marine Vessel's Electrical Power System: From its Birth to Present Day, (2015).

4. M.R. Patel, Shipboard Electrical Power Systems, CRC Press, 2016.

5. O. Alnes, S. Eriksen, B.-J. Vartdal, IEEE Electrific. Mag. 5 (3) 10-21. (2017)

6. G. Misyris, A. Marinopoulos, D. Doukas, T. Dimitris, P. Labridis, Electric Power Systems Research, 151, 115-124. (2017)

7. Balsamo F, Lauria D, Capasso C, Veneri O, Pede G. IEEE AEIT International Annual Conference, 1-6. (2016)

8. G. Schirripa Spagnolo, D. Papalilo, A. Martocchia, 10th International Conference on Environment and Electrical Engineering (EEEIC), IEEE, 1-4. (2011)

9. Oberhokamp, F. Diesel-electric propulsion concepts. How to match environmental and economical challenges (2007)

10. M. Chai, D. Reddy Bonthapalle, L. Sobrayen, S.K. Panda, D. Wu, X. Chen, Appl. Energy 231 747-756. (2018)

11. L. Bergh, U. Helldén. Electrical systems in pod propulsion. Master of Science Thesis of Electric Power Engineering. Göteborg, Sweden, 2007.

12. A. Haseltalab, M.A. Botto R. R. Negenborn, IFAC-PapersOnLine, 51 (29), 341-347. (2018)

13. M. Belkhayat, IEEE Electrific. Mag. 5 (3) 86-88. (2017)

14. H. Lan, S. Wen, Y. Hong, D. C. Yu, L. Zhang, Applied Energy 158 26-34. (2015)

15. A.J. Sorensen, R. Skjetne, T. Bo, M.R. Miyazaki, T.A. Johansen, I.B. Utne, E. Pedersen, IEEE Electrific. Mag. 5 (3) 68-73. (2017)

16. O. Mo, G. Guidi, 2018 IEEE Transportation Electrification Conference and Expo (ITEC), IEEE, 537-544. (2018)

17. E. Skjong, T.A. Johansen, M. Molinas, A.J. Sørensen, IEEE Trans. Transport. Electrific. 3 (1) 22-35. (2017)

18. S. Ahmed, A. Castellazzi, A. Williams, Trans. Environ. Electr. Eng. 1 (4) 37-43. (2016) 
19. C. Bordin, O. Mo, Including power management strategies and load profiles in the mathematical optimization of energy storage sizing for fuel consumption reduction in maritime vessels

20. B. Zahedi, L.E. Norum, K.B. Ludvigsen. J Power Sources 255 341-354. (2014)

21. Yan C, Venayagamoorthy GK, Corzine K a. Optimal location and sizing of energy storage modules for a smart electric ship power system. IEEE SSCI 2011 - Symp Ser Comput Intell - CIASG 20112011 IEEE Symp Comput Intell Appl Smart Grid 2011. p. 123-30. http://dx.doi.org/10.1109/CIASG.2011. 5953336.

22. Ericsen, T., Hingorani, N., Khersonsky, Y., 2006. Power electronics and future marine electrical systems. Ind. Appl. IEEE Trans. 42 (1), 155e163. http:// dx.doi.org/10.1109/TIA.2005.861306. 